\title{
Effective harmonic oscillator description of anharmonic molecular vibrations $^{\dagger}$
}

\author{
TAPTA KANCHAN ROY and M DURGA PRASAD* \\ School of Chemistry, University of Hyderabad, Hyderabad 500046 \\ e-mail: mdpsc@uohyd.ernet.in
}

\begin{abstract}
The validity of an effective harmonic oscillator approximation for anharmonic molecular vibrations is tested and compared with vibrational self consistent field and vibrational configurational interaction results. The effective harmonic oscillator is constructed variationally, by taking the trial wave function as a harmonic oscillator eigenfunction with the centroid and width parameter as variational paraeters. It is found that the effective harmonic oscillator approximation provides a description of the anharmonic eigenstates very similar to the vibrational self consistent field results. Coriolis coupling is also included in these studies.
\end{abstract}

Keywords. Effective harmonic oscillator; self-consistent field; vibrational states.

\section{Introduction}

Several methods have been developed to solve the Schrödinger equation for anharmonic molecular vibrations. Prominent among them is the vibrational self consistent field (VSCF) approach. ${ }^{1-12}$ Recently Gerber and co-workers described a procedure to accelerate the correlation corrected VSCF calculations for large molecules. ${ }^{13}$ Bowman and co-workers have developed three programs for carrying out such VSCF calculations. ${ }^{14-16}$ Most of these earlier works do not incorporate the Coriolis coupling terms in the self consistent field (SCF) procedure. Carter et $a l^{15}$ and more recently Rauhut ${ }^{17}$ discussed the possibilities of including the Coriolis coupling in the SCF procedure itself.

The VSCF approximation is based on a separable ansatz. The N-mode trial wave function is parameterized as

$$
\Psi\left(q_{1}, q_{2} \ldots . q_{n}\right)=\prod_{i} \Phi_{n_{i}}^{i}\left(q_{i}\right)
$$

where the one mode wave functions $\Phi_{n_{i}}^{i}$ are called the modals. In a typical calculation each of the modal is expanded in a basis

$$
\Phi_{n}^{i}=\sum_{m} \chi_{m}^{i} C_{m n}
$$

\footnotetext{
${ }^{\dagger}$ Dedicated to the memory of the late Professor S K Rangarajan

*For correspondence
}

The basis functions are usually orthogonal harmonic oscillator (HO) eigenfunctions. These functions are normally centered at the equilibrium point of the potential and the widths of the $\mathrm{HO}$ eigenfunction are chosen to match the zeroeth order frequencies of the potential. ${ }^{9,18}$

The goal of the present work is two-fold. First, we explore the possibility of describing vibrational states by a variationally optimized effective $\mathrm{HO}$ eigenfunctions. Since most of the VSCF calculations are carried out in $\mathrm{HO}$ basis, this study ought to provide an insight into reliability of such basis. The VSCF calculation themselves are carried out in the variationally optimized harmonic oscillator basis (VOHB). Second, we assess the importance of the Coriolis coupling at the level of SCF procedure itself for non-rotating states.

The VSCF equations in the presence of Coriolis coupling are presented in Section 2 and the contruction of VOHB is presented in Section 3. After a brief discussion of the program in Section 4 some illustrative results are presented in Section 5.

\section{The VSCF theory including Coriolis coupling}

In mass weighted normal coordinate representation, the Watson Hamiltonian ${ }^{19}$ for a non-rotating $(J=0)$ molecule is

$$
H=\frac{1}{2} \sum_{i=1}^{N} P_{i}^{2}-\frac{\hbar^{2}}{8} \sum \mu_{\alpha \alpha}+\frac{1}{2} \sum_{\alpha \beta} \mu_{\alpha \beta} \pi_{\alpha} \pi_{\beta}+V(Q) .
$$


Here, the first term is the pure vibrational kinetic energy term. The second one is the so called Watson term. The indices $\alpha$ and $\beta$ refer to the $x, y$ and $z$ components of Cartesian coordinates, and $\mu_{\alpha \beta}$ are the components of the inverse effective moment of inertia tensor at a given point on the potential energy surface (PES). $\pi_{\alpha}$ is the $\alpha^{\text {th }}$ component of the vibrational angular momentum and is given by

$$
\pi_{\alpha}=\sum_{i>j} \xi_{i j}^{\alpha}\left(Q_{i} P_{j}-Q_{j} P_{i}\right),
$$

where $\xi_{i j}^{\alpha}$ are the Coriolis coupling constants. Two approximations are made in defining the parameters of the Hamiltonian (3) in our calculations. First, the potential is taken to be a quartic polynomial in $Q_{i}$,

$$
\begin{aligned}
V(Q)= & \sum_{i} f_{i i} Q_{i}^{2}+\sum_{i \leq j \leq k} f_{i j k} Q_{i} Q_{j} Q_{k} \\
& +\sum_{i \leq j \leq k \leq l} f_{i j k l} Q_{i} Q_{j} Q_{k} Q_{l} .
\end{aligned}
$$

Second, following the earlier works, ${ }^{9,20}$ the moment of inertia is taken to be a constant. As stated earlier, a Hartree product of single-mode wave functions is taken as VSCF variational ansatz. Minimization of the total energy with respect to the modal functions $\Phi_{n_{i}}^{i}\left(Q_{i}\right)$ of (1) gives the working equations. The general form of these equations for mode $i$ is

$$
\left(h_{i}^{v s c f}-\varepsilon_{n_{i}}^{(i)}\right) \Phi_{n_{i}}^{i}\left(Q_{i}\right)=0
$$

where the $h_{i}^{\text {vscf }}$ is given by

$$
h_{i}^{\text {vscf }}=-\frac{1}{2 m_{i}}\left(\frac{\mathrm{d}^{2}}{\mathrm{~d} Q_{i}^{2}}\right)+\sum_{n=1}^{4} u_{i}^{(n)} Q_{i}^{n}+u_{0} .
$$

Here the effective mass $m_{i}$ is given by the equation

$$
m_{i}^{-1}=\left[1+2 \sum_{j} \zeta_{j i j i}\left\langle Q_{j}^{2}\right\rangle+2 \sum_{j \leq k} \zeta_{j i k i}\left\langle Q_{j}\right\rangle\left\langle Q_{k}\right\rangle\right],
$$

and the $\zeta$ matrix is defined as

$$
\zeta_{i j k l}=\sum_{\alpha} \xi_{i j}^{\alpha} I_{\alpha \alpha}^{-1} \xi_{k l}^{\alpha},
$$

where $I_{\alpha \alpha}$ are the moments of inertia along the principle axes of the molecule. Note that the effective mass in (8) appears due to Coriolis coupling. Since the Coriolis coupling term is a product of both posi- tion and momentum operators [cf., (3-4)], its partial averages lead to an effective kinetic energy operator and an additional quadratic potential term to the SCF Hamiltonian. The additional kinetic energy operator manifests itself in the form of an effective mass term in the SCF Hamiltonian. This effective mass goes to 1 in the absence of Coriolis coupling. The coefficients $u_{i}^{(n)}$ are the coefficients of $Q_{i}^{n}$ in the SCF potential,

$$
\begin{aligned}
u_{i}^{(0)}= & \frac{1}{2} \sum_{j} \zeta_{i j j i}, \\
u_{i}^{(1)}= & \sum_{j<k} f_{i j k}\left\langle Q_{j}\right\rangle\left\langle Q_{k}\right\rangle+\sum_{j} f_{i j j}\left\langle Q_{j}^{2}\right\rangle \\
& +\sum_{j<k<l} f_{i j k i}\left\langle Q_{j}\right\rangle\left\langle Q_{k}\right\rangle\left\langle Q_{l}\right\rangle+\sum_{j \neq k} f_{i j j k}\left\langle Q_{j}^{2}\right\rangle\left\langle Q_{k}\right\rangle \\
& +\sum_{j} f_{i j j j}\left\langle Q_{j}^{3}\right\rangle+\sum_{j, k} \zeta_{i j k j}\left\langle P_{j}^{2}\right\rangle\left\langle Q_{j}\right\rangle \\
u_{i}^{(2)}= & f_{i i}+\sum_{j} f_{i i j}\left\langle Q_{j}\right\rangle+\sum_{j} f_{i j j j}\left\langle Q_{j}^{2}\right\rangle \\
& +\sum_{j<k} f_{i i j k}\left\langle Q_{j}\right\rangle\left\langle Q_{k}\right\rangle+\sum_{j} \zeta_{i j i j}\left\langle P_{j}^{2}\right\rangle \\
u_{i}^{(3)}= & f_{i i i}+\sum_{j} f_{i i j j}\left\langle Q_{j}\right\rangle, \\
u_{i}^{(4)}= & f_{i i i i} .
\end{aligned}
$$

Since the expectation values $\left\langle Q_{i}^{n}\right\rangle$ and hence the SCF Hamiltonian in (6-14), depend on the modal functions $\Phi_{n}^{i}$, these equations have to be solved iteratively until self consistency is reached.

\section{Variationally optimized effective harmonic oscillator approximation}

We next turn to the variational formulation of the effective harmonic oscillator (EHO). Note that the EHO approximation is a special case of the VSCF approximation. The ansatz in this case is a harmonic oscillator eigenfunction. Such HO eigenfunctions are characterized by two parameters, the centroid of the function $\left(Q^{0}\right)$ and the width parameter $(\omega)$ of the functions,

$$
\chi_{n}^{i}(q)=h_{n}\left[\sqrt{m \omega}\left(Q-Q^{0}\right)\right] \exp \left[-m \omega\left(Q-Q^{0}\right)^{2} / 2\right] .
$$


Thus we posit a trial wave function for the $\mathrm{N}$-mode system as in (1), but write $\Phi_{n}^{i}$ as

$$
\Phi_{n}^{i}=\chi_{n}^{i}
$$

The working equation for $Q_{i}^{0}$ and $\omega_{i}$ are obtained by minimizing the resulting expectation value of the Hamiltonian with respect to $Q_{i}^{0}$ and $\omega_{i}$. The resulting equation for $Q_{i}^{0}$ is

$$
Q_{i}^{0}=\frac{1}{2 f_{i i}}\left[\begin{array}{l}
\sum_{j} f_{i j} g_{i j}\left(2 n_{j}+1\right) / 2 \omega_{j} \\
+\sum_{j \neq k} f_{i j k k} g_{i j k}\left(2 n_{j}+1\right) Q_{k}^{0} / 2 \omega_{j} \\
+\sum_{i<k} f_{i j k} g_{i j k} Q_{k}^{0} Q_{j}^{0}+\sum_{j \neq k \neq l} f_{i j k l} g_{i j k l} Q_{k}^{0} Q_{j}^{0} Q_{l}^{0} \\
+\sum_{i j k} \zeta_{i j k j}\left(2 n_{j}+1\right) Q_{k}^{0} \omega_{j}
\end{array}\right],
$$

and the equation for the corresponding frequency is

$$
m_{i} \omega_{i}=2\left[\begin{array}{c}
f_{i i}+\sum_{j} f_{i i j} g_{i j} Q_{j}^{0}+\sum_{j<k} f_{i j j k} g_{i j k} Q_{j}^{0} Q_{k}^{0} \\
+f_{i i i i} \frac{6 n_{i}^{2}+6 n_{i}+3}{\left(2 n_{i}+1\right) m_{i} \omega_{i}} \\
-\sum_{j i k i} \zeta_{j i k i} Q_{j}^{0} Q_{k}^{0}\left(m_{i} \omega_{i}\right)^{2} \\
+\sum_{j \neq i} f_{i i j j} g_{i j} \frac{\left(2 n_{j}+1\right)}{m_{j} \omega_{j}} \\
+\frac{1}{2} \sum_{j \neq i} \zeta_{i j i j}\left(2 n_{j}+1\right) \frac{m_{j}^{2} \omega_{j}^{2}-m_{i}^{2} \omega_{i}^{2}}{m_{j} \omega_{j}}
\end{array}\right]^{1 / 2},
$$

where

$$
m_{i}^{-1}=1+2 \sum_{j i k i} \zeta_{j k i} Q_{j}^{0} Q_{k}^{0}+\sum_{i j i j} \zeta_{i j i j} \sum_{i j i j} \frac{2 n_{j}+1}{m_{j} \omega_{j}} .
$$

Here $n_{i}$ is the occupation number of the $i^{\text {th }}$ mode and $g_{i j}$ and $g_{i j k}$ are the combinatorial factors associated with the expansion of the potential around $Q_{i}^{0}$. These equations are on the one hand, generalization of the Hartree approximation used by several earlier authors to describe the ground state of an anharmonic oscillator $^{21-23}$ to arbitrary state, and, on the other hand, special case of (10-14). Once $Q_{i}^{0}$ and $\omega_{i}$ are determined, the eigenfunctions of the EHO

$$
h_{i}^{E H O}=-\frac{1}{2 m_{i}} \frac{\mathrm{d}^{2}}{\mathrm{~d} Q_{i}^{2}}+\frac{1}{2} m_{i} \omega_{i}^{2}\left(Q_{i}-Q_{i}^{0}\right)^{2},
$$

become the modals for the corresponding vibrational state. The state energy is given by the expectation value of the Hamiltonian over the target state. Since the EHO approximation is a special case of the VSCF approximation in that it constrains the modal functions to be $\mathrm{HO}$ eigenfunctions, the $\mathrm{EHO}$ energy for the ground state would always be higher than the VSCF ground states. However, this may not hold always for the excited states, due to variational collapse. ${ }^{24}$

\section{Description of program}

The VSCF-VOHB program is based on the theory presented above. The program requires the quartic force field in the mass weighted normal coordinates as input. It first reads the number of vibrational modes, the number of irreducible representations in molecular symmetry group and a row matrix containing the number of normal coordinates belonging to a given irreducible representation in sequence. Symmetry is used to determine the nonzero elements of the potential energy function, and is used to the level of the largest abelian point group, $D_{2 h}$. The program then reads the details of the force field. The unique set of non-zero $f$-coefficients that define the quartic force field in (5) are read. These are stored in a one-dimensional array. The program next reads the Coriolis coupling constants $\xi_{i j}$ followed by the number of basis functions to be used in the SCF calculation for each mode and the quantum numbers of the target state.

After initializing the data it does the EHO calculation described in Section 3 and determines the parameters $Q_{i}^{0}$ and $\omega_{i}$. It then shifts the origin of the coordinate system to $Q_{i}^{0}$ and recalculates the potential and Coriolis coupling parameters with respect to this new origin. This step is required to simplify the calculation of the matrix elements of the various operators that appear in the Hamiltonian. These matrix elements are evaluated analytically on the fly as and when required. The coefficient matrix of the modal functions is initialized to identity matrix and the SCF procedure is started. The expectation values of various operators that contribute to the effective single particle potential are constructed. In the next step the SCF Hamiltonian matrix elements are calculated in the EHO basis. The matrices are then diagonalized to obtain the modals and the associated single particle state energies. The process is iterated until the zeroeth order SCF energy is converged to, within a preset tolerance. At this point the first order 
correction to energy is calculated to obtain the final SCF energy. The code is available in source form upon request to the authors.

\section{Results and discussions}

We have used VSCF-VOHB program to calculate the vibrational energy levels of formaldehyde. The

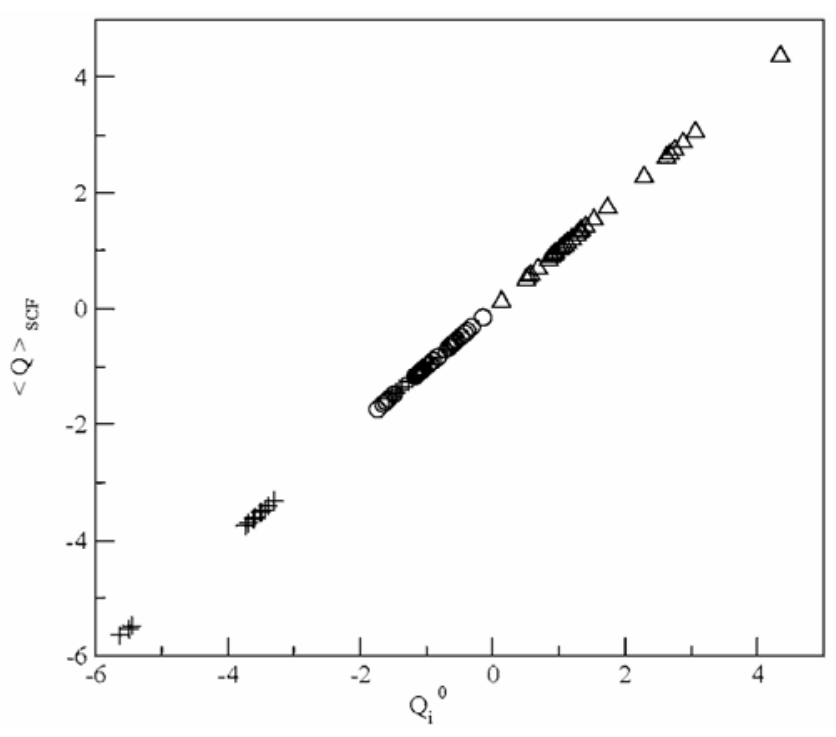

Figure 1. $\left\langle Q_{i}\right\rangle_{\mathrm{SCF}}$ vS $\mathrm{Q}_{i}^{0}$ (in a.u.) for the three totally symmetric modes for formaldehyde (+: mode $1, \Delta$ : mode 2 and $\mathrm{O}$ : mode 3 ).

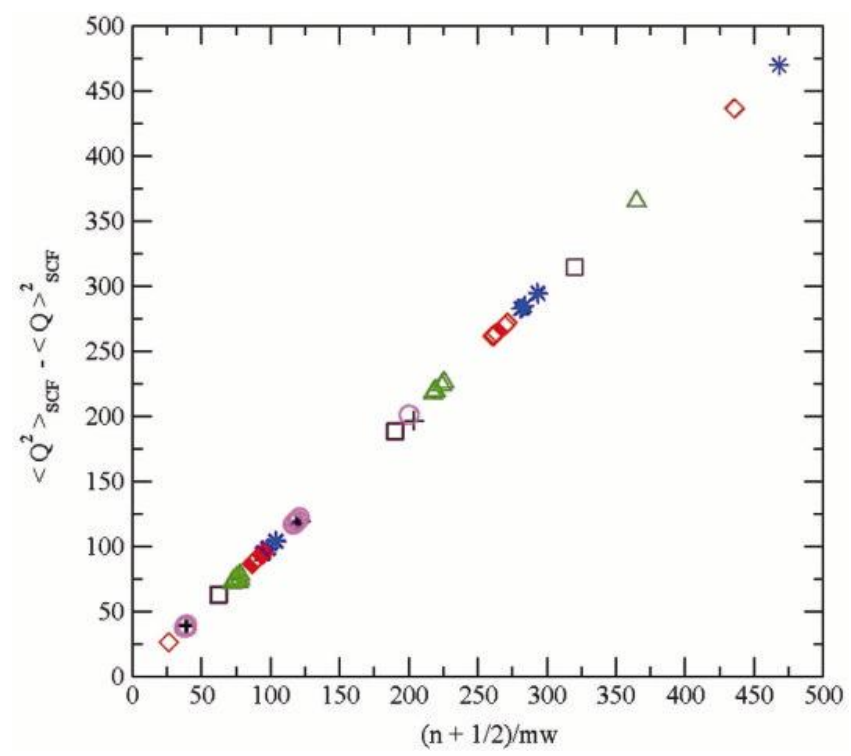

Figure 2. $\left(\left\langle Q^{2}\right\rangle_{\mathrm{SCF}}-\langle Q\rangle_{\mathrm{SCF}}^{2}\right)$ vs $(\mathrm{n}+1 / 2) / m \omega$ (in a.u.) for formaldehyde (+: mode $1, \square$ : Mode 2, $\Delta$ : mode 3 , *: mode $4, \mathrm{O}$ : mode 5 and $\diamond$ : mode 6 ). potential energy and Coriolis coupling parameters were taken from ref. 9. The first point we discuss is the quality of the EHO eigenfunctions. Specifically the expectation values of $\left\langle Q_{i}\right\rangle$ and $\left\langle Q_{i}^{2}\right\rangle$ for the reference EHO states and the final SCF states are very close. Within the EHO approximation, the expectation value of the displacement is just $Q_{i}^{0}$. We plot $\left\langle Q_{i}\right\rangle_{\mathrm{SCF}}$ vs $Q_{i}^{0}$ for the three totally symmetric normal modes for all the states containing up to 2 quanta of energy in figure 1 . As can be seen the agreement between $Q_{i}^{0}$ and $\left\langle Q_{i}\right\rangle_{\mathrm{SCF}}$ is excellent. The largest deviation is less than $1 \%$.

The expectation value of $(Q-\langle Q\rangle)^{2}$ in the EHO approximation is given by $(n+1 / 2) / m \omega$. We plot $\left\langle(Q-\langle Q\rangle)^{2}\right\rangle_{\mathrm{SCF}}$ against the corresponding value in the EHO approximation in figure 2. The largest deviation is around $4 \%$ for the $1_{2}$ state.

We next look at the overlap between the EHO eigenstates and the corresponding SCF states. This is plotted as the function of the SCF state energy in figure 3 . As can be seen the reference wave function has an overlap of almost 1 in most of the cases. The maximum deviation occurs for the $1_{2}$ state which has an overlap of 0.9 .

Given that the first two moments of the EHO states and the corresponding SCF states are almost identical and the overlap of the two functions is close to 1, it may be expected that the EHO energy would be close to the SCF energy. This data is presented in figure 4. As can be seen the SCF energy correlates almost linearly with the EHO energy with a slope of almost $1 \cdot 0$.

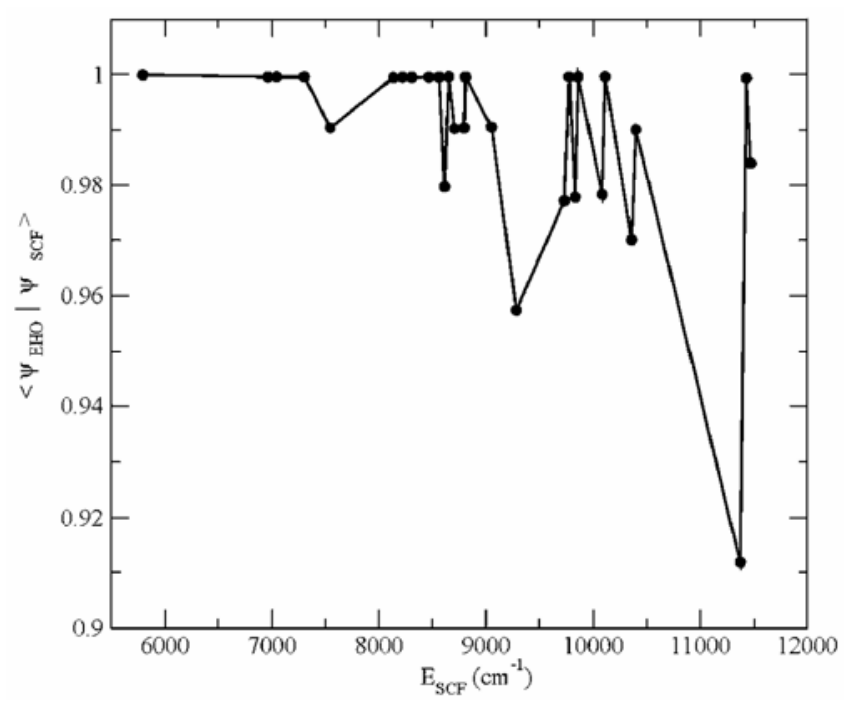

Figure 3. Overlap of the reference EHO eigenfunction with the SCF function vs. the state energy. 
Table 1. SCF and EHO energies with and without Coriolis coupling $\left(\mathrm{in} \mathrm{cm}^{-1}\right)$.

\begin{tabular}{lrcccr}
\hline State & $E_{\text {coriolis }}^{\mathrm{SCF}}$ & $E_{\text {coriolis }}^{\mathrm{SCF}}-E_{\text {no-coriolis }}^{\mathrm{SCF}}$ & $E_{\text {coriolis }}^{\mathrm{EHO}}$ & $E_{\text {coriolis }}^{\mathrm{EHO}}-E_{\text {no-coriolis }}^{\mathrm{EHO}}$ & ${ }^{\mathrm{a}} E_{\text {coriolis }}^{\mathrm{CI}}$ \\
\hline $4_{1}$ & $6960 \cdot 8$ & $13 \cdot 08$ & $6966 \cdot 68$ & $13 \cdot 01$ & $6937 \cdot 8$ \\
$5_{1}$ & $8650 \cdot 6$ & $10 \cdot 57$ & $8655 \cdot 82$ & $10 \cdot 65$ & $8635 \cdot 0$ \\
$4_{2}$ & $8134 \cdot 0$ & $26 \cdot 88$ & $8140 \cdot 15$ & $26 \cdot 71$ & $8092 \cdot 3$ \\
$5_{2}$ & $11431 \cdot 5$ & $22 \cdot 80$ & $11436 \cdot 0$ & $23 \cdot 2$ & $11476 \cdot 6$ \\
$4_{1} 5_{1}$ & $9776 \cdot 9$ & $44 \cdot 44$ & $9782 \cdot 37$ & $44 \cdot 32$ & $9777 \cdot 5$ \\
\hline
\end{tabular}

${ }^{\mathrm{a}}$ From ref. 9

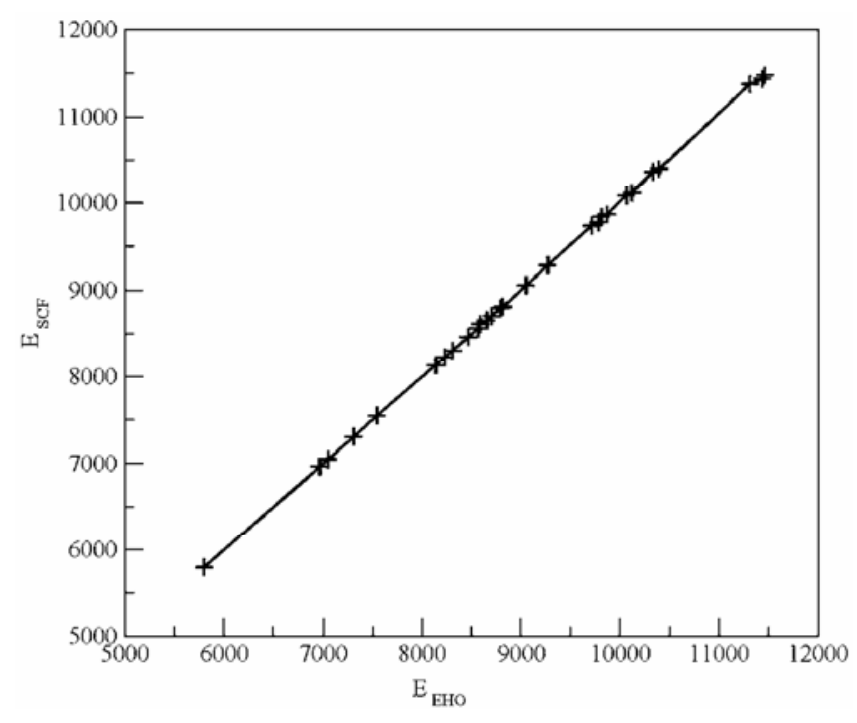

Figure 4. $E_{\mathrm{SCF}} \mathrm{vs} E_{\mathrm{EHO}}\left(\right.$ in $\left.\mathrm{cm}^{-1}\right)$.

Indeed, it appears that the VSCF modals are essentially the variationally optimized EHO eigenfunctions. The data presented above indicates that the EHO eigenfunctions resemble the SCF modals both in the energy domain and in the coordinate space to a significant extent. Given this close resemblance between the two sets of function we expect that the VOHB would provide a good basis for post SCF calculations such as VCI and VCCM as well as the VSCF basis. This would be particularly convenient because the anharmonic matrix elements are available in analytical form for the $\mathrm{HO}$ basis, and most of them are zeroes. Such is not the case with the VSCF basis. So, one might expect a faster code in the EHO basis for the post SCF calculations.

We next turn to the relative importance of Coriolis coupling. For the large molecules this term is insignificant because it is inversely proportional to the moment of inertia. For small molecules, however, it could be significant. For formaldehyde, modes 4 and 5 are significantly affected by Coriolis coupling. In table 1 we present the energies of a few states involving these two modes. As can be seen the contribution of Coriolis coupling to the SCF energy is of the same order of magnitude as the correlation energy, typically of the order of a few tens of wave numbers. The relative sign of the two terms, however, is random. Thus it is difficult to pass a judgement on whether the Coriolis coupling should be included at the SCF level or not with the limited data available at this stage. More studies on this aspect are desirable. The EHO approximation is able to capture the effect of the Coriolis coupling more or less fully.

\section{Acknowledgements}

T K Roy acknowledges Council of Scientific and Industrial Research (CSIR), New Delhi, for a sustaining Fellowship. University of Grants Commission (UGC) is thanked for infrastructure development through the UPE program to the University of Hyderabad and through the CAS program to the School of Chemistry. Computational facilities provided by the Center for Modelling Simulation and Design (CMSD) of the University of Hyderabad are acknowledged.

\section{References}

1. Carney G C, Sprandel L L and Kern W C $1978 A d v$. Chem. Phys. 27305

2. Bowman J M 1978 J. Chem. Phys. 68608

3. Bowman J M, Chirstoffel K M and Tobin F L $1979 \mathrm{~J}$. Phys. Chem. 83905

4. Bowman J M 1986 Acc. Chem. Res. 19202

5. Ratner M A and Gerber R B 1986 J. Phys. Chem. 90 20

6. Roth R M, Gerber R B and Ratner M S 1983 J. Phys. Chem. 872376

7. Bowman J M, Chirstoffel K and Tobin F J $1979 \mathrm{~J}$. Phys. Chem. 83905

8. Garrett B C and Truhlar D G 1982 Chem. Phys. Lett. 9264 
9. Romanowski H and Bowman J M $1984 \mathrm{~J}$. Chem. Phys. 824155

10. Tobin F L and Bowman J M 1979 Chem. Phys. 47 151

11. Smith A D, Liu W K and Noid D W 1984 Chem. Phys. 89345

12. Roitberg A, Gerber R B and Ratner M A 1995 Science 2681318

13. Pele L, Brauner B and Gerber R B 2007 Theor. Chem. Acc. 11769

14. Ramanowski $\mathrm{H}$ and Bowman M J 1985 POLYMODE (QCPE 496) QCPE Bull. 564

15. Carter S, Bowman J M and Handy N C 1998 Theor. Chem. Acta 100191
16. Jelski D A, Hally R H and Bowman J M $1996 \mathrm{~J}$. Comp. Chem. 171645

17. Rauhut G 2004 J. Chem. Phys. 1219313

18. Romanowski H and Bowman J M 1984 Chem. Phys. Lett. 110235

19. Watson J K G 1968 Mol. Phys. 15479

20. Sibert III E L 1988 J. Chem. Phys. 88 4378; Sibert III E L 1989 J. Chem. Phys. 902672

21. Nagalakshmi V, Lakshminarayana V, Sumitra G and Prasad M Durga 1994 Chem. Phys. Lett. 217279

22. Bishop R F and Flynn M F 1988 Phys. Rev. A38 2211

23. Hsue C S and Chern J L 1984 Phys. Rev. D29 643

24. Epstein S T 1974 Variation method in quantum chemistry (New York: Academic Press) 